\title{
Exponential polynomials with Fatou and non-escaping sets of finite Lebesgue measure
}

\author{
MAREIKE WOLFF \\ Mathematisches Seminar, Christian-Albrechts-Universität zu Kiel, \\ Ludewig-Meyn-Str. 4, D-24118 Kiel, Germany \\ (e-mail:wolff@math.uni-kiel.de)
}

(Received 14 August 2019 and accepted in revised form 2 October 2020)

Abstract. We give conditions ensuring that the Fatou set and the complement of the fast escaping set of an exponential polynomial $f$ both have finite Lebesgue measure. Essentially, these conditions are designed such that $|f(z)| \geq \exp \left(|z|^{\alpha}\right)$ for some $\alpha>0$ and all $z$ outside a set of finite Lebesgue measure.

Key words: exponential polynomial, fast escaping set, Fatou set, Julia set, Lebesgue measure

2020 Mathematics Subject Classification: 37F10 (Primary); 30D05 (Secondary)

\section{Introduction and results}

Let $f$ be a transcendental entire function, and let $f^{n}$ denote the $n$th iterate of $f$. The Fatou set $F(f)$ of $f$ is the set of all $z \in \mathbb{C}$ such that the iterates $\left(f^{n}\right)_{n \in \mathbb{N}}$ form a normal family in a neighbourhood of $z$, and the Julia set $J(f)$ is the complement of $F(f)$. These sets have an important role in complex dynamics. Clearly, $F(f)$ is open, and $J(f)$ is closed. Moreover, $J(f)$ is always non-empty, and either $J(f)=\mathbb{C}$ or $J(f)$ has empty interior. An introduction to the dynamics of transcendental entire functions can be found in [2]. The escaping set of $f$ is defined by

$$
I(f):=\left\{z: f^{n}(z) \rightarrow \infty \text { as } n \rightarrow \infty\right\} .
$$

Eremenko [7] showed that $I(f)$ is always non-empty, and that $J(f)=\partial I(f)$. For $r>0$, let $M(r, f):=\max _{|z|=r}|f(z)|$ denote the maximum modulus of $f$, and let $M^{n}(r, f)$ be its $n$th iterate with respect to $r$. The fast escaping set $A(f)$ is a subset of the escaping set. It was introduced by Bergweiler and Hinkkanen [5] and is defined by

$$
A(f):=\left\{z: \text { there exists } l \in \mathbb{N} \text { such that }\left|f^{n}(z)\right| \geq M^{n-l}(R, f) \text { for } n>l\right\},
$$


where $R$ is chosen such that $M(r, f)>r$ for $r \geq R$. For a thorough discussion of the fast escaping set, see [12].

We are interested in the Lebesgue measure of the sets defined above. McMullen [9] showed that the Julia set of $f(z)=\sin (a z+b), a \neq 0$, has positive Lebesgue measure. In fact, it can be seen from the proof that $J(f) \cap A(f)$ also has positive measure. Sixsmith [14] proved that if $f(z)=\sum_{j=1}^{q} a_{j} \exp \left(\omega_{q}^{j} z\right)$, where $q \geq 2, a_{j} \in \mathbb{C} \backslash\{0\}$, and $\omega_{q}=\exp (2 \pi i / q)$, then $J(f) \cap A(f)$ has positive measure. Sixsmith remarked without proof that his result remains true for

$$
f(z)=\sum_{j=1}^{q} a_{j} \exp \left(b_{j} z\right),
$$

where $q \geq 3, a_{j}, b_{j} \in \mathbb{C} \backslash\{0\}, \arg \left(b_{j}\right)<\arg \left(b_{j+1}\right)<\arg \left(b_{j}\right)+\pi$ for $j \in\{1, \ldots, q-1\}$, and $\arg \left(b_{q}\right)>\arg \left(b_{1}\right)+\pi$, with the argument chosen in $[0,2 \pi)$. Bergweiler and Chyzhykov [4] gave conditions ensuring that the Julia set and the escaping set of a transcendental entire function of completely regular growth have positive measure. These conditions are satisfied for the functions (1). In fact, they are also satisfied if one allows $\arg \left(b_{j+1}\right)=\arg \left(b_{j}\right)+\pi$ for some $j \in\{1, \ldots, q-1\}$ or $\arg \left(b_{q}\right)=\arg \left(b_{1}\right)+\pi$. Further criteria for Julia sets and (fast) escaping sets to have positive measure are given in $[1,3]$.

For certain functions, it is possible to obtain stronger results in the sense that one can bound the size of the complement of the Julia set or (fast) escaping set. Schubert [13] used McMullen's methods to prove that if $f(z)=\sinh (z)$, then the Lebesgue measure of $F(f)$ and $\mathbb{C} \backslash I(f)$ is finite in any horizontal strip of width $2 \pi$. In fact, the proof shows that one may replace $I(f)$ by the fast escaping set $A(f)$ here. Schubert's result was generalized by Zhang and Yang [15] to functions of the form $f(z)=P\left(e^{z}\right) / e^{z}$, where $P$ is a polynomial of degree at least two satisfying $P(0) \neq 0$.

There seem to be no papers whose main aim is to show that the Lebesgue measure of the Fatou set or the complement of the (fast) escaping set of certain transcendental entire functions is finite. However, there are some results presented in papers mainly treating a different subject. We mention two of them. Hemke [8, Theorem 5.1] showed that if

$$
f(z)=Q_{1}(z) \exp (P(z))+Q_{2}(z) \exp (-P(z)),
$$

where $P, Q_{1}, Q_{2}$ are polynomials with $Q_{1}, Q_{2} \not \equiv 0$ and $\operatorname{deg}(P) \geq 3$, then the Lebesgue measure of $\mathbb{C} \backslash I(f)$ is finite. One example of such a function is $f(z)=\sin \left(z^{3}\right)$. A result of Bock [6, Example 2] says that if $f(z)=\sin (\pi z)$, then $F(f)=\emptyset$, and $\left(f^{n}(z)\right)$ tends to infinity for almost all $z \in \mathbb{C}$.

This is different for $f(z)=\sin (z)$, which is conjugate to the function $\sinh (z)$ considered by Schubert, and $f(z)=\sin \left(z^{2}\right)$.

Example 1.1. The Fatou set and the non-escaping set of $\sin (z)$ and $\sin \left(z^{2}\right)$ have infinite Lebesgue measure.

We will verify this in $\S 5$. See Figure 1 for an illustration of the non-escaping sets of $\sin (z)$, $\sin \left(z^{2}\right)$ and $\sin \left(z^{3}\right)$. 

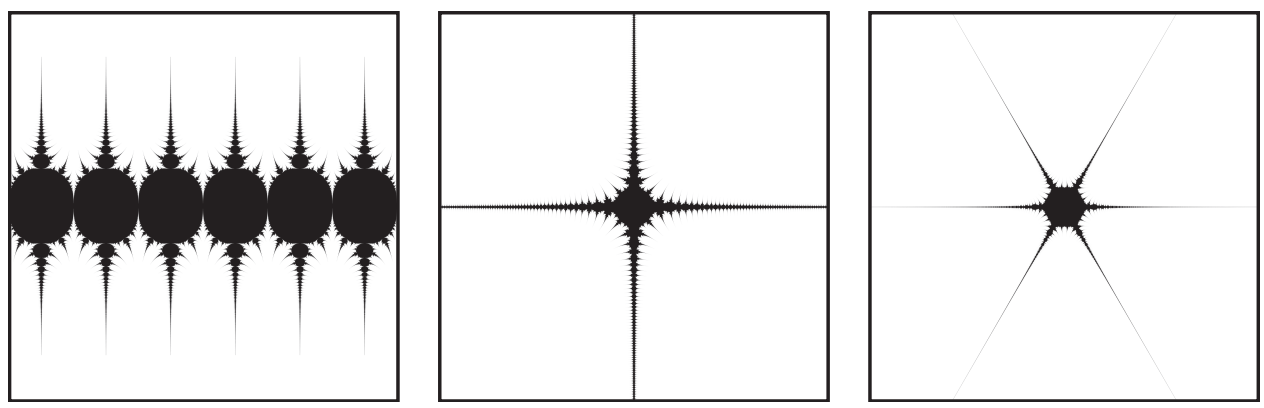

FIGURE 1. The non-escaping sets of $\sin (z), \sin \left(z^{2}\right)$ and $\sin \left(z^{3}\right)$.

In this paper, we consider exponential polynomials of the form

$$
f(z)=\sum_{j=1}^{N} Q_{j}(z) \exp \left(b_{j} z^{d}+P_{j}(z)\right),
$$

where $Q_{j}$ and $P_{j}$ are polynomials with $\operatorname{deg}\left(P_{j}\right)<d$. We give conditions ensuring that the Lebesgue measure of the complement of $J(f) \cap A(f)$ is finite.

THEOREM 1.2. Let

$$
f(z):=\sum_{j=1}^{N} Q_{j}(z) \exp \left(b_{j} z^{d}+P_{j}(z)\right),
$$

where $d \in \mathbb{N}$ with $d \geq 3, P_{j}$ and $Q_{j}$ are polynomials with $Q_{j} \not \equiv 0$ and $\operatorname{deg}\left(P_{j}\right)<d$, and $b_{j} \in \mathbb{C} \backslash\{0\}$ are distinct numbers satisfying $\arg \left(b_{j}\right) \leq \arg \left(b_{j+1}\right)<\arg \left(b_{j}\right)+\pi$ for all $j \in\{1, \ldots, N-1\}$ and $\arg \left(b_{N}\right)>\arg \left(b_{1}\right)+\pi$, with the argument chosen in $[0,2 \pi)$. Then the Lebesgue measure of $\mathbb{C} \backslash(A(f) \cap J(f))$ is finite.

Note that the conditions on the $b_{j}$ imply that $N \geq 3$. Recall that Sixsmith's result for the functions (1) remains true if $\arg \left(b_{j+1}\right)=\arg \left(b_{j}\right)+\pi$ for some $j \in\{1, \ldots, q-1\}$ or $\arg \left(b_{q}\right)=\arg \left(b_{1}\right)+\pi$. This is not true in general for Theorem 1.2, as the following example shows.

Example 1.3. Let

$$
h(z):=\frac{1}{2} \exp \left(z^{3}+i z\right)-\frac{1}{2} \exp \left(-z^{3}+i z\right)=\exp (i z) \sinh \left(z^{3}\right) .
$$

Then $h$ has a superattracting fixed point at zero, and the attractive basin of zero has infinite Lebesgue measure. In particular, the Lebesgue measure of $\mathbb{C} \backslash(A(h) \cap J(h))$ is infinite.

We will verify this in $\$ 5$. However, the next theorem yields that under additional assumptions on the polynomials $P_{j}$, Theorem 1.2 can be extended to the case where $\arg \left(b_{j+1}\right)=\arg \left(b_{j}\right)+\pi$ for some $j \in\{1, \ldots, N-1\}$ or $\arg \left(b_{N}\right)=\arg \left(b_{1}\right)+\pi$. These assumptions are satisfied if $\operatorname{deg}\left(P_{j}-\left(b_{j} / b_{j+1}\right) P_{j+1}\right) \leq d-3$ or $\operatorname{deg}\left(P_{1}-\right.$ $\left.\left(b_{1} / b_{N}\right) P_{N}\right) \leq d-3$, respectively. We actually state a slightly more general condition, taking into account that several of the numbers $b_{j}$ may have the same argument. 
THEOREM 1.4. Let

$$
f(z):=\sum_{j=1}^{N} Q_{j}(z) \exp \left(b_{j} z^{d}+P_{j}(z)\right),
$$

where $d \in \mathbb{N}$ with $d \geq 3, P_{j}$ and $Q_{j}$ are polynomials with $Q_{j} \not \equiv 0$ and $\operatorname{deg}\left(P_{j}\right)<d$, and $b_{j} \in \mathbb{C} \backslash\{0\}$ are distinct numbers satisfying $\arg \left(b_{j}\right) \leq \arg \left(b_{j+1}\right) \leq \arg \left(b_{j}\right)+\pi$ for all $j \in\{1, \ldots, N-1\}$ and $\arg \left(b_{N}\right) \geq \arg \left(b_{1}\right)+\pi$, with the argument chosen in $[0,2 \pi)$.

If there exists $j \in\{1, \ldots, N-1\}$ such that $\arg \left(b_{j+1}\right)=\arg \left(b_{j}\right)+\pi$, or if $\arg \left(b_{N}\right)=$ $\arg \left(b_{1}\right)+\pi$, in addition suppose that there are $k, l \in\{1, \ldots, N\}$ with $\arg \left(b_{k}\right)=\arg \left(b_{j}\right)$ and $\arg \left(b_{l}\right)=\arg \left(b_{j+1}\right)$, or $\arg \left(b_{k}\right)=\arg \left(b_{1}\right)$ and $\arg \left(b_{l}\right)=\arg \left(b_{N}\right)$, respectively, such that

$$
\operatorname{deg}\left(P_{k}-\frac{b_{k}}{b_{l}} P_{l}\right) \leq d-3 .
$$

Then the Lebesgue measure of $\mathbb{C} \backslash(A(f) \cap J(f))$ is finite.

Note that the conditions on the $b_{j}$ imply that $N \geq 2$. Theorem 1.2 is a special case of Theorem 1.4. Also, the functions (2) considered by Hemke satisfy the assumptions of Theorem 1.4.

Throughout the rest of the paper, let $f$ be an entire function satisfying the assumptions of Theorem 1.4. In $\S 2$, we will show that $f$ can be approximated by simpler functions in large parts of the complex plane, and use this to prove that $|f(z)|$ is large outside a set of finite measure. Then, in $\S 3$, we show that $f$ is injective in certain small disks. We finish the proof of Theorem 1.4 in $\S 4$, using a construction similar to one that was used in McMullen's paper [9] and has since then been used by various authors. Finally, in §5, we verify the properties of Example 1.1 and Example 1.3.

\section{The behaviour of $f$}

In this section, we prove several properties of the function $f$. We first introduce some notation. For $j, k \in\{1, \ldots, N\}$ with $j \neq k$, let

$$
P_{j, k}(z):=\left(b_{j} z^{d}+P_{j}(z)\right)-\left(b_{k} z^{d}+P_{k}(z)\right)=\left(b_{j}-b_{k}\right) z^{d}+\left(P_{j}(z)-P_{k}(z)\right) .
$$

Let

$$
v:=d-\frac{5}{2}
$$

and define the sets

$$
U_{1}:=\left\{w \in \mathbb{C}:|\operatorname{Re}(w)|<|w|^{\nu / d}\right\}
$$

and

$$
U_{2}:=\left\{w \in \mathbb{C}:|\operatorname{Re}(w)|<2|w|^{\nu / d}\right\} .
$$

Moreover, define 'exceptional sets'

$$
E_{l}:=\bigcup_{\substack{j, k=1 \\ j \neq k}}^{N} P_{j, k}^{-1}\left(U_{l}\right)
$$




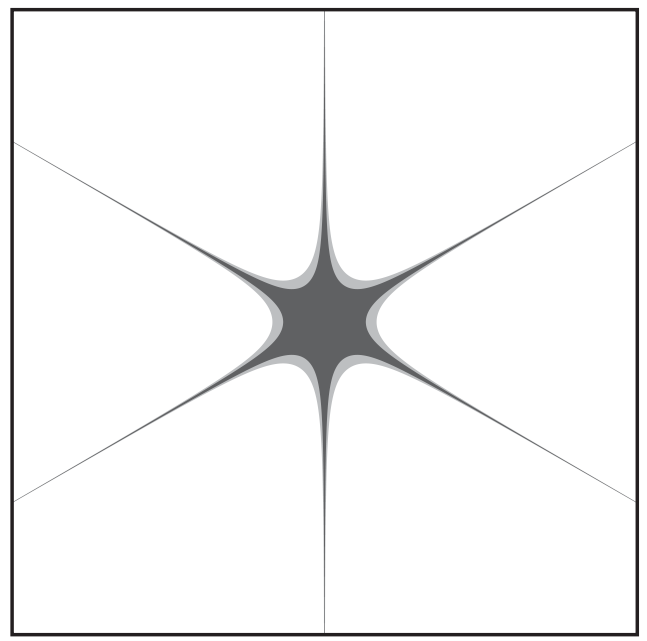

FIgURE 2. The sets $E_{1}$ (dark grey) and $E_{2}$ (light and dark grey) for $f(z)=Q_{1}(z) \exp \left(z^{3}\right)+Q_{2}(z) \exp \left(-z^{3}\right)$.

for $l \in\{1,2\}$ (see Figure 2). We will later prove that the function $f$ behaves 'nicely' outside $E_{1}$.

For $z_{0} \in \mathbb{C}$ and $r>0$, we denote by $D\left(z_{0}, r\right):=\left\{z:\left|z-z_{0}\right|<r\right\}$ the open disk of radius $r$ around $z_{0}$. Moreover, let meas $(A)$ denote the Lebesgue measure of a measurable set $A \subset \mathbb{C}$.

Lemma 2.1. Let $P$ be a polynomial of degree $d$, with $d$ as before. Then the Lebesgue measure of $P^{-1}\left(U_{2}\right)$ is finite.

Proof. Write

$$
P(z)=\sum_{j=0}^{d} a_{j} z^{j}
$$

Fix $R>2^{2 d / 5}$ such that all critical values of $P$ are contained in $D(0, R)$, and let $V$ be a component of $P^{-1}(\mathbb{C} \backslash(\overline{D(0, R)} \cup(-\infty, 0]))$. Then the restriction $\left.P\right|_{V}: V \rightarrow$ $\mathbb{C} \backslash(\overline{D(0, R)} \cup(-\infty, 0])$ is biholomorphic. Let $\varphi$ denote the corresponding inverse function. Then $P^{-1}\left(U_{2}\right) \cap V=\varphi\left(U_{2} \backslash \overline{D(0, R)}\right)$. For $\eta(r):=\arcsin \left(2 r^{-5 /(2 d)}\right)$, let

$$
W:=U_{2} \backslash \overline{D(0, R)}=\left\{r e^{i \theta}: r>R, \min \left\{\left|\theta-\frac{\pi}{2}\right|,\left|\theta-\frac{3 \pi}{2}\right|\right\}<\eta(r)\right\} .
$$

We have

$$
\begin{aligned}
& \operatorname{meas}\left(P^{-1}\left(U_{2}\right) \cap V\right)=\int_{W}\left|\varphi^{\prime}(z)\right|^{2} d x d y=\int_{W} \frac{1}{\left|P^{\prime}(\varphi(z))\right|^{2}} d x d y \\
& =\int_{R}^{\infty} r\left(\int_{\pi / 2-\eta(r)}^{\pi / 2+\eta(r)} \frac{1}{\left|P^{\prime}\left(\varphi\left(r e^{i \theta}\right)\right)\right|^{2}} d \theta+\int_{3 \pi / 2-\eta(r)}^{3 \pi / 2+\eta(r)} \frac{1}{\left|P^{\prime}\left(\varphi\left(r e^{i \theta}\right)\right)\right|^{2}} d \theta\right) d r
\end{aligned}
$$


If $r$ is sufficiently large, then

$$
r=\left|P\left(\varphi\left(r e^{i \theta}\right)\right)\right| \leq 2^{d /(d-1)}\left|a_{d}\right|\left|\varphi\left(r e^{i \theta}\right)\right|^{d} .
$$

Thus,

$$
\left|\varphi\left(r e^{i \theta}\right)\right| \geq 2^{-1 /(d-1)}\left|a_{d}\right|^{-1 / d} \cdot r^{1 / d}
$$

and

$$
\left|P^{\prime}\left(\varphi\left(r e^{i \theta}\right)\right)\right| \geq \frac{1}{2} d\left|a_{d}\right|\left|\varphi\left(r e^{i \theta}\right)\right|^{d-1} \geq \frac{1}{4} d\left|a_{d}\right|^{1 / d} \cdot r^{(d-1) / d} .
$$

Since $\arcsin (x) \leq(\pi / 2) x$ for $0 \leq x \leq 1$, we also have

$$
\eta(r)=\arcsin \left(2 r^{-5 /(2 d)}\right) \leq \pi r^{-5 /(2 d)} .
$$

Using (4) and (5), we get

$$
\begin{aligned}
\operatorname{meas}\left(P^{-1}\left(U_{2}\right) \cap V\right) & \leq \int_{R}^{\infty} r \cdot 4 \eta(r) \cdot \frac{16}{d^{2}\left|a_{d}\right|^{2 / d} r^{2(d-1) / d}} d r \\
& \leq \frac{64 \pi}{d^{2}}\left|a_{d}\right|^{-2 / d} \int_{R}^{\infty} r \cdot r^{-5 /(2 d)} \cdot r^{-2(d-1) / d} d r \\
& =\frac{64 \pi}{d^{2}}\left|a_{d}\right|^{-2 / d} \int_{R}^{\infty} r^{-(1+1 /(2 d))} d r<\infty
\end{aligned}
$$

Since there are only finitely many such components $V$, and also $P^{-1}(\overline{D(0, R)})$ has finite Lebesgue measure, the claim follows.

The following corollary is an immediate consequence of Lemma 2.1.

COROLlARY 2.2. The Lebesgue measure of $E_{1}$ and $E_{2}$ is finite.

The next Lemma yields that if $R_{0}>0$ is large, then in each component of $\mathbb{C} \backslash\left(E_{1} \cup\right.$ $\left.D\left(0, R_{0}\right)\right), f$ behaves like one of its summands $Q_{m}(z) \exp \left(b_{m} z^{d}+P_{m}(z)\right)$.

LEMMA 2.3. Let $\varepsilon>0$. Then there exists $R_{0}>0$ such that for each connected component $V$ of $\mathbb{C} \backslash\left(E_{1} \cup D\left(0, R_{0}\right)\right)$ there is an $m \in\{1, \ldots, N\}$ such that for all $z \in V$ and all $j \in$ $\{1, \ldots, N\}$ with $j \neq m$, we have

$$
\operatorname{Re}\left(b_{m} z^{d}+P_{m}(z)\right)>\operatorname{Re}\left(b_{j} z^{d}+P_{j}(z)\right)
$$

and

$$
\begin{gathered}
\left|\frac{f(z)}{Q_{m}(z) \exp \left(b_{m} z^{d}+P_{m}(z)\right)}-1\right|<\varepsilon, \\
\left|\frac{f^{\prime}(z)}{d b_{m} z^{d-1} Q_{m}(z) \exp \left(b_{m} z^{d}+P_{m}(z)\right)}-1\right|<\varepsilon, \\
\left|\frac{f^{\prime \prime}(z)}{d^{2} b_{m}^{2} z^{2 d-2} Q_{m}(z) \exp \left(b_{m} z^{d}+P_{m}(z)\right)}-1\right|<\varepsilon .
\end{gathered}
$$

Proof. Let $R_{0}>0$ such that all zeros of the polynomials $P_{j, k}$ are contained in $D\left(0, R_{0}\right)$, and let $V$ be a component of $\mathbb{C} \backslash\left(E_{1} \cup D\left(0, R_{0}\right)\right)$. Let $z_{0} \in V$, and let $m \in\{1, \ldots, N\}$ 
such that

$$
\operatorname{Re}\left(b_{m} z_{0}^{d}+P_{m}\left(z_{0}\right)\right)=\max _{1 \leq j \leq N} \operatorname{Re}\left(b_{j} z_{0}^{d}+P_{j}\left(z_{0}\right)\right) .
$$

Let $j \in\{1, \ldots, N\}$ with $j \neq m$. For all $z \in V$, we have $P_{m, j}(z) \notin U_{1}$ and hence

$$
\left|\operatorname{Re}\left(b_{m} z^{d}+P_{m}(z)\right)-\operatorname{Re}\left(b_{j} z^{d}+P_{j}(z)\right)\right|=\left|\operatorname{Re}\left(P_{m, j}(z)\right)\right| \geq\left|P_{m, j}(z)\right|^{v / d} .
$$

In particular,

$$
\operatorname{Re}\left(b_{m} z^{d}+P_{m}(z)\right)-\operatorname{Re}\left(b_{j} z^{d}+P_{j}(z)\right) \neq 0
$$

for all $z \in V$. By continuity and (6), we get

$$
\operatorname{Re}\left(b_{m} z^{d}+P_{m}(z)\right)-\operatorname{Re}\left(b_{j} z^{d}+P_{j}(z)\right)>0
$$

for all $z \in V$, and hence

$$
\operatorname{Re}\left(b_{m} z^{d}+P_{m}(z)\right)-\operatorname{Re}\left(b_{j} z^{d}+P_{j}(z)\right) \geq\left|P_{m, j}(z)\right|^{v / d}
$$

by (7). Thus, for all $z \in V$,

$$
\begin{aligned}
& \left|\frac{f(z)}{Q_{m}(z) \exp \left(b_{m} z^{d}+P_{m}(z)\right)}-1\right| \\
& =\left|\frac{\sum_{j=1}^{N} Q_{j}(z) \exp \left(b_{j} z^{d}+P_{j}(z)\right)-Q_{m}(z) \exp \left(b_{m} z^{d}+P_{m}(z)\right)}{Q_{m}(z) \exp \left(b_{m} z^{d}+P_{m}(z)\right)}\right| \\
& =\left|\sum_{\substack{j=1 \\
j \neq m}}^{N} \frac{Q_{j}(z)}{Q_{m}(z)} \exp \left(b_{j} z^{d}+P_{j}(z)-\left(b_{m} z^{d}+P_{m}(z)\right)\right)\right| \\
& \leq \sum_{\substack{j=1 \\
j \neq m}}^{N}\left|\frac{Q_{j}(z)}{Q_{m}(z)}\right| \exp \left(\operatorname{Re}\left(b_{j} z^{d}+P_{j}(z)\right)-\operatorname{Re}\left(b_{m} z^{d}+P_{m}(z)\right)\right) \\
& \quad \leq \sum_{\substack{j=1 \\
j \neq m}}^{N}\left|\frac{Q_{j}(z)}{Q_{m}(z)}\right| \exp \left(-\left|P_{j, m}(z)\right|^{v / d}\right)<\varepsilon
\end{aligned}
$$

if $R_{0}$, and hence $|z|$ is sufficiently large. This is the result for $f$.

Moreover,

$$
f^{\prime}(z)=\sum_{j=1}^{N}\left(d b_{j} z^{d-1} Q_{j}(z)+P_{j}^{\prime}(z) Q_{j}(z)+Q_{j}^{\prime}(z)\right) \exp \left(b_{j} z^{d}+P_{j}(z)\right) .
$$

The result for $f^{\prime}$ now follows from similar estimates as above and the fact that

$$
d b_{j} z^{d-1} Q_{j}(z)+P_{j}^{\prime}(z) Q_{j}(z)+Q_{j}^{\prime}(z)=(1+o(1)) d b_{j} z^{d-1} Q_{j}(z)
$$

as $|z| \rightarrow \infty$. Analogously, the result for $f^{\prime \prime}$ follows from

$$
f^{\prime \prime}(z)=\sum_{j=1}^{N}(1+o(1)) d^{2} b_{j}^{2} z^{2 d-2} Q_{j}(z) \exp \left(b_{j} z^{d}+P_{j}(z)\right)
$$

as $|z| \rightarrow \infty$. 
Remark. In order to prove Lemma 2.3, we did not need any assumptions on the arguments of the $b_{j}$. In particular, the statement remains true without the additional condition (3) in the case where $\arg \left(b_{j+1}\right)=\arg \left(b_{j}\right)+\pi$ for some $j \in\{1, \ldots, N-1\}$ or $\arg \left(b_{N}\right)=$ $\arg \left(b_{1}\right)+\pi$. This is different for the next result.

Lemma 2.4. Let $\alpha \in(0, v)$. Then there exists $R_{1}>0$ such that for all $z \in \mathbb{C} \backslash E_{1}$ with $|z| \geq R_{1}$, we have

$$
|f(z)| \geq \exp \left(|z|^{\alpha}\right) \quad \text { and } \quad\left|f^{\prime}(z)\right| \geq \exp \left(|z|^{\alpha}\right) .
$$

Remark. Without the additional condition (3) in the case where $\arg \left(b_{j+1}\right)=\arg \left(b_{j}\right)+\pi$ for some $j \in\{1, \ldots, N\}$ or $\arg \left(b_{N}\right)=\arg \left(b_{1}\right)+\pi$, the statement of Lemma 2.4 is not true in general. We will prove in $\S 5$ that the function $h(z)=(1 / 2) \exp \left(z^{3}+i z\right)-$ $(1 / 2) \exp \left(-z^{3}+i z\right)$ given in Example 1.3 is bounded in a set of infinite Lebesgue measure.

Proof of Lemma 2.4. We prove the statement for $f$. The proof for $f^{\prime}$ is analogous. Let $z \in \mathbb{C} \backslash E_{1}$. If $|z|$ is sufficiently large, then by Lemma 2.3 there is $m \in\{1, \ldots, N\}$ such that

$$
\operatorname{Re}\left(b_{m} z^{d}+P_{m}(z)\right)>\operatorname{Re}\left(b_{j} z^{d}+P_{j}(z)\right)
$$

for all $j \in\{1, \ldots, N\}$ with $j \neq m$ and

$$
\left|\frac{f(z)}{Q_{m}(z) \exp \left(b_{m} z^{d}+P_{m}(z)\right)}-1\right| \leq \frac{1}{2} .
$$

Then

$$
|f(z)| \geq \frac{1}{2}\left|Q_{m}(z)\right| \exp \left(\operatorname{Re}\left(b_{m} z^{d}+P_{m}(z)\right)\right) .
$$

Thus, it suffices to show that there exists $j \in\{1, \ldots, N\}$ with

$$
\operatorname{Re}\left(b_{j} z^{d}+P_{j}(z)\right) \geq 2|z|^{\alpha} .
$$

We first consider the case where $f$ satisfies the assumptions of Theorem 1.2, that is,

$$
\arg \left(b_{j+1}\right)<\arg \left(b_{j}\right)+\pi \text { for all } j \in\{1, \ldots, N-1\} \text { and } \arg \left(b_{N}\right)>\arg \left(b_{1}\right)+\pi .
$$

Then there is a constant $C>0$ such that for all $w \in \mathbb{C}$ there exists $j \in\{1, \ldots, N\}$ with $\operatorname{Re}\left(b_{j} w^{d}\right) \geq 2 C|w|^{d}$. In particular, this applies to $w=z$. Since $\operatorname{deg}\left(P_{j}\right)<d$, we get

$$
\operatorname{Re}\left(b_{j} z^{d}+P_{j}(z)\right) \geq 2 C|z|^{d}-\left|P_{j}(z)\right| \geq C|z|^{d}>2|z|^{\alpha}
$$

if $|z|$ is sufficiently large.

Now suppose that $f$ does not satisfy the assumptions of Theorem 1.2. Then the assumptions of Theorem 1.4 imply that there are $k, l \in\{1, \ldots, N\}$ satisfying $\mid \arg \left(b_{k}\right)-$ $\arg \left(b_{l}\right) \mid=\pi$, such that $\operatorname{deg}\left(P_{k}-\left(b_{k} / b_{l}\right) P_{l}\right) \leq d-3$. Thus, there exist polynomials $g, g_{k}, g_{l}$ with $\operatorname{deg}(g) \leq d-1$ and $\max \left\{\operatorname{deg}\left(g_{k}\right), \operatorname{deg}\left(g_{l}\right)\right\} \leq d-3$ such that

$$
P_{k}=b_{k} g+g_{k} \quad \text { and } \quad P_{l}=b_{l} g+g_{l} .
$$

With $\beta_{k}:=\arg \left(b_{k}\right)$, we have

$$
b_{k}=\left|b_{k}\right| e^{i \beta_{k}} \quad \text { and } \quad b_{l}=-\left|b_{l}\right| e^{i \beta_{k}}
$$


Thus,

$$
b_{k}-b_{l}=\left(\left|b_{k}\right|+\left|b_{l}\right|\right) e^{i \beta_{k}}
$$

and

$$
P_{k, l}(z)=\left(\left|b_{k}\right|+\left|b_{l}\right|\right) e^{i \beta_{k}}\left(z^{d}+g(z)\right)+\left(g_{k}(z)-g_{l}(z)\right) .
$$

Moreover, we assume without loss of generality that

$$
\operatorname{Re}\left(b_{k}\left(z^{d}+g(z)\right)\right) \geq \operatorname{Re}\left(b_{l}\left(z^{d}+g(z)\right)\right) .
$$

Then $\operatorname{Re}\left(b_{k}\left(z^{d}+g(z)\right)\right) \geq 0$. Since $z \notin E_{1}$, we get

$$
\left|P_{k, l}(z)\right|^{\nu / d} \leq\left|\operatorname{Re}\left(P_{k, l}(z)\right)\right| \leq\left(\left|b_{k}\right|+\left|b_{l}\right|\right) \operatorname{Re}\left(e^{i \beta_{k}}\left(z^{d}+g(z)\right)\right)+\left|g_{k}(z)-g_{l}(z)\right| .
$$

Hence,

$$
\begin{aligned}
\operatorname{Re}\left(b_{k} z^{d}+P_{k}(z)\right) & =\operatorname{Re}\left(b_{k}\left(z^{d}+g(z)\right)+g_{k}(z)\right) \\
& =\left|b_{k}\right| \operatorname{Re}\left(e^{i \beta_{k}}\left(z^{d}+g(z)\right)\right)+\operatorname{Re}\left(g_{k}(z)\right) \\
& \geq \frac{\left|b_{k}\right|}{\left|b_{k}\right|+\left|b_{l}\right|}\left(\left|P_{k, l}(z)\right|^{\nu / d}-\left|g_{k}(z)-g_{l}(z)\right|\right)-\left|g_{k}(z)\right| .
\end{aligned}
$$

Since

$$
\left|P_{k, l}(z)\right| \geq 2^{-d / v}\left(\left|b_{k}\right|+\left|b_{l}\right|\right)|z|^{d}
$$

if $|z|$ is large, and since $\max \left\{\operatorname{deg}\left(g_{k}\right), \operatorname{deg}\left(g_{l}\right)\right\}<v$, we deduce that

$$
\operatorname{Re}\left(b_{k} z^{d}+P_{k}(z)\right) \geq \frac{1}{4}\left|b_{k}\right|\left(\left|b_{k}\right|+\left|b_{l}\right|\right)^{(v / d)-1}|z|^{\nu}>2|z|^{\alpha}
$$

if $|z|$ is sufficiently large. This completes the proof.

\section{Injectivity}

The aim of this section is to prove that $f$ is injective in certain disks contained in $\mathbb{C} \backslash E_{1}$.

We start with a basic injectivity criterion (see, e.g., [11, Proposition 1.10]).

Lemma 3.1. Let $D \subset \mathbb{C}$ be a convex domain, and let $h: D \rightarrow \mathbb{C}$ be holomorphic. If $\operatorname{Re}\left(h^{\prime}(z)\right)>0$ for all $z \in D$, then $h$ is injective in $D$.

We also require the following criterion.

Lemma 3.2. Let $z_{0} \in \mathbb{C}$ and $r>0$. Let $h$ be holomorphic in $D\left(z_{0}, r\right)$. Suppose that $h^{\prime}(\zeta) \neq 0$ for all $\zeta \in D\left(z_{0}, r\right)$ and

$$
\sup _{\left|\zeta-z_{0}\right|<r}\left|\frac{h^{\prime \prime}(\zeta)}{h^{\prime}(\zeta)}\right|<\frac{1}{r} .
$$

Then $h$ is injective in $D\left(z_{0}, r\right)$.

This follows directly from Becker's univalence criterion (see, e.g., [10, Theorem 6.7]). However, Lemma 3.2 may also be proved by much more elementary arguments using Lemma 3.1. We sketch the proof here. 
Sketch of proof. We may assume without loss of generality that $h^{\prime}\left(z_{0}\right)=1$. Let $\psi$ be the branch of $\log h^{\prime}$ in $D\left(z_{0}, r\right)$ satisfying $\psi\left(z_{0}\right)=0$. Then, for all $z \in D\left(z_{0}, r\right)$,

$$
|\psi(z)|=\left|\int_{z_{0}}^{z} \frac{h^{\prime \prime}(\zeta)}{h^{\prime}(\zeta)} d \zeta\right|<\frac{1}{r}\left|z-z_{0}\right|<1
$$

Thus, $\arg \left(h^{\prime}(z)\right)=\operatorname{Im}(\psi(z)) \in(-1,1)$. In particular, $\operatorname{Re}\left(h^{\prime}(z)\right)>0$. Hence, $h$ is injective in $D\left(z_{0}, r\right)$ by Lemma 3.1 .

We now state the main result of this section.

Lemma 3.3. Let $\sigma \in\left(0,1 /\left(4 d \max _{j}\left|b_{j}\right|\right)\right)$. Then there exists $R_{2}>0$ such that the following holds. If $z \in \mathbb{C} \backslash E_{1}$ with $|z| \geq R_{2}$ is such that $D\left(z, 2 \sigma|z|^{-(d-1)}\right) \subset \mathbb{C} \backslash E_{1}$, then $f$ is injective in $D\left(z, 2 \sigma|z|^{-(d-1)}\right)$.

Proof. Let $r:=2 \sigma|z|^{-(d-1)}$. By Lemma 2.3, there exists $m \in\{1, \ldots, N\}$ such that

$$
\sup _{|\zeta-z|<r}\left|\frac{f^{\prime \prime}(\zeta)}{f^{\prime}(\zeta)}\right| \leq \frac{3}{2} d\left|b_{m}\right| \sup _{|\zeta-z|<r}|\zeta|^{d-1} \leq 2 d\left|b_{m}\right||z|^{d-1}<\frac{1}{r}
$$

if $|z|$ is sufficiently large. Thus, $f$ is injective in $D(z, r)$ by Lemma 3.2.

For $z \in \mathbb{C}$ and $A \subset \mathbb{C}$, let $\operatorname{dist}(z, A):=\inf \{|z-a|: a \in A\}$ denote the Euclidean distance between $z$ and $A$.

Lemma 3.4. There are $C_{1}, R_{3}>0$ such that if $z \in \mathbb{C} \backslash E_{2}$ and $|z| \geq R_{3}$, then

$$
\operatorname{dist}\left(z, E_{1}\right) \geq C_{1}|z|^{-3 / 2} .
$$

The following corollary is an immediate consequence of Lemmas 3.4 and 3.3.

COROLlaRY 3.5. Let $\sigma$ be as in Lemma 3.3. There is $R_{4}>0$ such that if $z \in \mathbb{C} \backslash E_{2}$ and $|z| \geq R_{4}$, then $f$ is injective in $D\left(z, 2 \sigma|z|^{-(d-1)}\right)$.

Proof of Lemma 3.4. Let $z \in \mathbb{C} \backslash E_{2}$. It suffices to show that if $|z|$ is sufficiently large, then

$$
\operatorname{dist}\left(z, E_{1} \cap D(z, 1)\right) \geq C_{1}|z|^{-3 / 2}
$$

for some constant $C_{1}>0$. Let $w \in E_{1} \cap D(z, 1)$. Then there are $j, k \in\{1, \ldots, N\}$ with $j \neq k$ such that $\left|\operatorname{Re}\left(P_{j, k}(w)\right)\right| \leq\left|P_{j, k}(w)\right|^{v / d}$. If $|z|$ is sufficiently large, then $|w| \leq$ $(6 / 5)^{1 / v}|z|$ and

$$
\begin{aligned}
\left|P_{j, k}(z)-P_{j, k}(w)\right| & \geq \operatorname{Re}\left(P_{j, k}(z)\right)-\operatorname{Re}\left(P_{j, k}(w)\right) \\
& \geq 2\left|P_{j, k}(z)\right|^{\nu / d}-\left|P_{j, k}(w)\right|^{v / d} \\
& \geq 2 \cdot \frac{4}{5}\left|b_{j}-b_{k}\right|^{v / d}|z|^{v}-\frac{6}{5}\left|b_{j}-b_{k}\right|^{v / d}|w|^{v} \\
& \geq \frac{8}{5}\left|b_{j}-b_{k}\right|^{v / d}|z|^{\nu}-\left(\frac{6}{5}\right)^{2}\left|b_{j}-b_{k}\right|^{v / d}|z|^{v} \\
& =\frac{4}{25}\left|b_{j}-b_{k}\right|^{v / d}|z|^{d-5 / 2}
\end{aligned}
$$


On the other hand,

$$
\begin{aligned}
\left|P_{j, k}(z)-P_{j, k}(w)\right| & =\left|\int_{w}^{z} P_{j, k}^{\prime}(\zeta) d \zeta\right| \leq \sup _{\zeta \in D(z, 1)}\left|P_{j, k}^{\prime}(\zeta)\right| \cdot|z-w| \\
& \leq 2 d\left|b_{j}-b_{k}\right|(|z|+1)^{d-1}|z-w| \leq 4 d\left|b_{j}-b_{k}\right||z|^{d-1}|z-w|
\end{aligned}
$$

Thus,

$$
|z-w| \geq \frac{\left|b_{j}-b_{k}\right|^{v / d-1}}{25 d}|z|^{-3 / 2} \geq C_{1}|z|^{-3 / 2}
$$

for $C_{1}=\left(\min _{l \neq n}\left|b_{l}-b_{n}\right|^{v / d-1}\right) / 25 d$.

\section{Proof of Theorem 1.4}

In this section, we prove Theorem 1.4. First, we collect several results that we require. For $\alpha>0$, consider the function

$$
E_{\alpha}:[0, \infty) \rightarrow[0, \infty), \quad E_{\alpha}(x)=\exp \left(x^{\alpha}\right) .
$$

We will use the following result [3, Lemma 2.1].

LemMA 4.1. Let $\beta>\alpha>0$. Then there exists $x_{0}>0$ such that

$$
E_{\alpha}^{k}(x) \geq E_{\beta}^{k-2}(x) \quad \text { for all } k \geq 4 \text { and } x \geq x_{0} .
$$

The next lemma is due to Sixsmith [14, Theorem 3.1].

LEMMA 4.2. Let $h$ be a transcendental entire function and $z_{0} \in I(h)$. Let $z_{n}:=h^{n}\left(z_{0}\right)$ for all $n \in \mathbb{N}$. Suppose that there exist $\lambda>1$ and $N \geq 0$ such that

$$
h\left(z_{n}\right) \neq 0 \text { and }\left|z_{n} \frac{h^{\prime}\left(z_{n}\right)}{h\left(z_{n}\right)}\right| \geq \lambda \quad \text { for all } n \geq N .
$$

Then either $z_{0}$ is in a multiply connected Fatou component of $h$, or $z_{0} \in J(h)$.

The following result is due to Zheng [16, Corollary 6 and Remark (J)].

LEMMA 4.3. Let $h$ be a transcendental entire function of the form

$$
h(z)=\sum_{j=1}^{M} q_{j}(z) \exp \left(p_{j}(z)\right)
$$

with polynomials $p_{j}$ and $q_{j}$. Then the Fatou set of $h$ has no multiply connected components.

For a curve $\gamma \subset \mathbb{C}$ we denote by $\ell(\gamma)$ the Euclidean length of $\gamma$.

Lemma 4.4. Let $\gamma \subset \mathbb{C}$ be a curve of positive length, and let $s \in(0, \ell(\gamma))$. Then

$$
\operatorname{meas}(\{z: \operatorname{dist}(z, \gamma) \leq s\}) \leq \frac{9 \pi}{2} s \cdot \ell(\gamma) .
$$

Proof. Let $L \in \mathbb{N}$ such that $L-1<\ell(\gamma) / s \leq L$. We divide $\gamma$ into $L$ subcurves $\gamma_{1}, \ldots, \gamma_{L}$ satisfying $\ell\left(\gamma_{j}\right) \leq s$ for all $j \in\{1, \ldots, L\}$. Then, for $j \in\{1, \ldots, L\}$, there 
is an $a_{j} \in \mathbb{C}$ such that $\gamma_{j} \subset \overline{D\left(a_{j}, s / 2\right)}$. We have

$$
\left\{z: \operatorname{dist}\left(z, \gamma_{j}\right) \leq s\right\} \subset \overline{D\left(a_{j},(3 / 2) s\right)} \text {. }
$$

Thus,

$$
\operatorname{meas}\left(\left\{z: \operatorname{dist}\left(z, \gamma_{j}\right) \leq s\right\}\right) \leq \frac{9 \pi}{4} s^{2} .
$$

Using $(\ell(\gamma) / s)+1 \leq 2 \ell(\gamma) / s$, we get

$$
\operatorname{meas}(\{z: \operatorname{dist}(z, \gamma) \leq s\}) \leq L \frac{9 \pi}{4} s^{2}<\left(\frac{\ell(\gamma)}{s}+1\right) \frac{9 \pi}{4} s^{2} \leq \frac{9 \pi}{2} s \cdot \ell(\gamma) .
$$

The next result is a direct consequence of the well-known Koebe distortion theorem (see, e.g., [10, Theorem 1.6]).

LEMMA 4.5. Let $z_{0} \in \mathbb{C}, r>0$ and $\rho \in(0,1)$. Suppose that $h: D\left(z_{0}, r\right) \rightarrow \mathbb{C}$ is holomorphic and injective. Then, for all $z \in D\left(z_{0}, \rho r\right)$,

$$
\frac{\left|h^{\prime}\left(z_{0}\right)\right|}{(1+\rho)^{2}} \leq \frac{\left|h(z)-h\left(z_{0}\right)\right|}{\left|z-z_{0}\right|} \leq \frac{\left|h^{\prime}\left(z_{0}\right)\right|}{(1-\rho)^{2}} .
$$

Moreover,

$$
\frac{\max _{|z| \leq \rho r}\left|h^{\prime}(z)\right|}{\min _{|z| \leq \rho r}\left|h^{\prime}(z)\right|} \leq\left(\frac{1+\rho}{1-\rho}\right)^{4}
$$

Proof of Theorem 1.4. Throughout the proof, write

$$
J_{A}:=J(f) \cap A(f) .
$$

The strategy of the proof is as follows. First, we construct a suitable collection $\mathcal{S}$ of squares in $\mathbb{C} \backslash E_{1}$ such that $\mathbb{C} \backslash \bigcup_{S \in \mathcal{S}} S$ has finite Lebesgue measure. For $S_{0} \in \mathcal{S}$, we then show that points in $S_{0}$ which stay in $\bigcup_{S \in \mathcal{S}} S$ under iteration lie in $J_{A}$, and that the set of such points has large density in $S_{0}$. Finally, we prove that $\sum_{S \in \mathcal{S}}$ meas $\left(S \backslash J_{A}\right)<\infty$.

Let $\varepsilon=1 / 3, \alpha \in(0, v), \sigma \in\left(0,1 /\left(4 d \max _{j}\left|b_{j}\right|\right)\right)$ and $\beta>d$, and let $R_{0}, R_{1}, R_{2}, x_{0}$ be the corresponding constants from Lemmas 2.3, 2.4, 3.3 and 4.1, respectively. Also, let $C_{1}, R_{3}$ be the constants from Lemma 3.4. Let $B_{0}$ be an open square centred at zero with sides parallel to the real and imaginary axes such that all $z \in \mathbb{C} \backslash B_{0}$ satisfy

$$
\begin{gathered}
|z|>\max \left\{R_{0}, \ldots, R_{3}, x_{0}\right\}, \\
\frac{C_{1}}{|z|^{3 / 2}}>\frac{3 \sigma}{|z|^{d-1}}, \\
M(|z|, f) \leq E_{\beta}(|z|), \\
\frac{1}{2} d \min _{k}\left|b_{k}\right||z|^{d}>2, \\
\operatorname{meas}\left(E_{2}\right) \leq\left(1-\frac{18 \pi \sigma}{\sqrt{2}|z|^{d-1}}\right) \exp \left(|z|^{\alpha}\right),
\end{gathered}
$$


and

$$
\left(\frac{5}{3}\right)^{4} \frac{32|z|^{2(d-2)}}{\sigma^{2} \exp \left(|z|^{\alpha}\right)} \leq \exp \left(-\frac{1}{2}|z|^{\alpha}\right) .
$$

For a compact set $X \subset \mathbb{C}$, let

$$
\mathcal{M}(X):=\max _{z \in X}|z| \quad \text { and } \quad \mu(X):=\min _{z \in X}|z| .
$$

More generally, for a continuous function $g: X \rightarrow \mathbb{C}$, let

$$
\mathcal{M}(X, g):=\max _{z \in X}|g(z)| \quad \text { and } \quad \mu(X, g):=\min _{z \in X}|g(z)| .
$$

Let $\tilde{\mathcal{S}}$ be a collection of closed squares in $\mathbb{C}$ with sides parallel to the real and imaginary axes such that:

- $\bigcup_{S \in \tilde{\mathcal{S}}} S=\mathbb{C} \backslash B_{0}$;

- $\quad$ for all $S_{1}, S_{2} \in \tilde{\mathcal{S}}$ with $S_{1} \neq S_{2}$, we have $S_{1}^{\circ} \cap S_{2}^{\circ}=\emptyset$; and

- $\quad$ for all $S \in \tilde{\mathcal{S}}$, the side length $s$ of $S$ satisfies

$$
\frac{\sigma}{4 \sqrt{2} \mu(S)^{d-1}} \leq s \leq \frac{\sigma}{\sqrt{2} \mathcal{M}(S)^{d-1}}
$$

If the side length of $B_{0}$ is sufficiently large, this can be achieved as follows. First, divide $\mathbb{C} \backslash B_{0}$ into squares of a fixed size so that the side length of all squares satisfies the lower bound. If the side length $s$ of a square does not satisfy the upper bound, divide it into four squares of side length $s / 2$, and then continue this procedure until the side length of the squares satisfies the upper bound.

Let $\mathcal{S}$ be the collection of all $S \in \tilde{\mathcal{S}}$ such that $\operatorname{dist}\left(S, E_{1}\right)>2 \sigma / \mu(S)^{d-1}$. By Lemma 3.4, the definition of $\mathcal{S}$, and (8),

$$
\mathbb{C} \backslash\left(E_{2} \cup B_{0}\right) \subset \bigcup_{S \in \mathcal{S}} S \subset \mathbb{C} \backslash\left(E_{1} \cup B_{0}\right) .
$$

Next, we construct a subset of $J_{A}$ as an intersection of nested sets. Fix a square $S_{0} \in \mathcal{S}$. Let

$$
\mathcal{K}_{0}:=\left\{S_{0}\right\}
$$

and, for $n \in \mathbb{N}$, let

$$
\mathcal{K}_{n}:=\left\{T_{n} \subset S_{0}: f^{n}\left(T_{n}\right) \in \mathcal{S} \text { and } T_{n} \subset T_{n-1} \text { for some } T_{n-1} \in \mathcal{K}_{n-1}\right\} .
$$

We first show that

$$
T:=\bigcap_{n=0}^{\infty}\left(\bigcup_{T_{n} \in \mathcal{K}_{n}} T_{n}\right) \subset J_{A} .
$$

To do so, let $z \in T$. Then $f^{n}(z) \in \mathbb{C} \backslash E_{1}$ for all $n \geq 0$. Using Lemma 2.4, Lemma 4.1 and (9), we get

$$
\left|f^{n}(z)\right| \geq E_{\alpha}^{n}(|z|) \geq E_{\beta}^{n-2}(|z|) \geq M^{n-2}(|z|, f)
$$




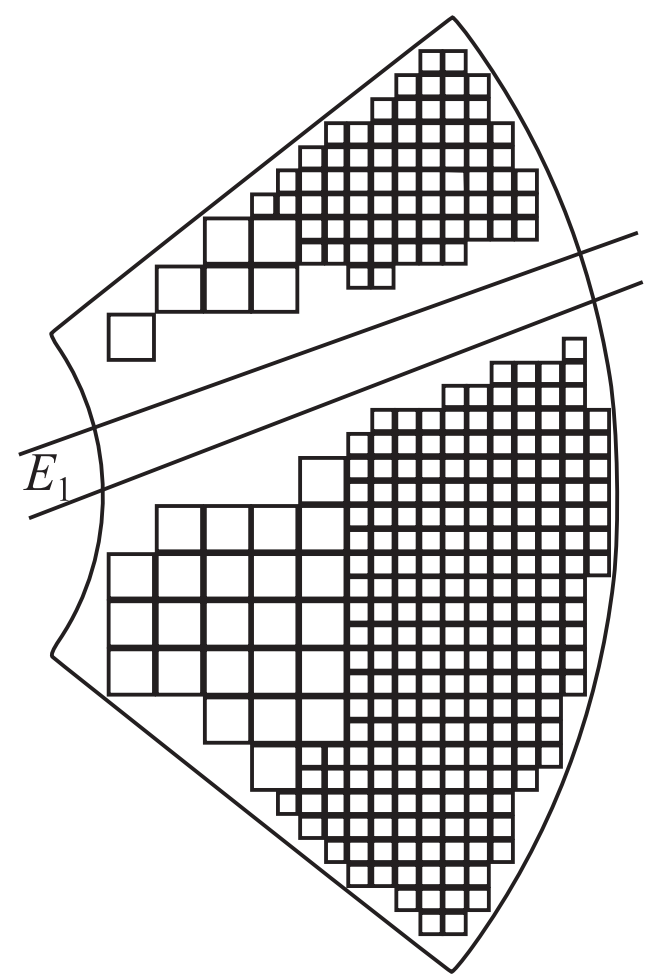

FIGURE 3. An illustration of $\operatorname{pack}(f(S))$ (not to scale). It consists of squares contained in $f(S)$ which are not too close to $E_{1}$. The size of the squares decreases as their distance from zero increases.

for all $n \geq 4$. This yields $z \in A(f)$. For $n \in \mathbb{N}$, let $z_{n}:=f^{n}(z)$. By Lemma 2.3, Lemma 2.4 and (10),

$$
\left|z_{n} \frac{f^{\prime}\left(z_{n}\right)}{f\left(z_{n}\right)}\right| \geq \frac{1}{2} d \min _{k}\left|b_{k}\right|\left|z_{n}\right|^{d}>2 .
$$

By Lemmas 4.2 and $4.3, z \in J(f)$. Thus, $T \subset J_{A}$.

For $A \subset \mathbb{C}$, define

$$
\operatorname{pack}(A):=\{S \in \mathcal{S}: S \subset A\} .
$$

Moreover, for measurable $A, B \subset \mathbb{C}$ with meas $(B)>0$, let

$$
\operatorname{dens}(A, B):=\frac{\operatorname{meas}(A \cap B)}{\operatorname{meas}(B)}
$$

denote the density of $A$ in $B$. We will show that for any square $S \in \mathcal{S}$,

$$
\operatorname{dens}\left(f(S) \backslash \bigcup_{S^{\prime} \in \operatorname{pack}(f(S))} S^{\prime}, f(S)\right) \leq \exp \left(-\frac{1}{2} \mu(S)^{\alpha}\right) .
$$

See Figure 3 for an illustration of $\operatorname{pack}(f(S))$. 
By Lemma 3.3, $f$ is injective in $S$. We have

$$
\operatorname{meas}(f(S))=\int_{S}\left|f^{\prime}(z)\right|^{2} d x d y \geq \operatorname{meas}(S) \cdot \mu\left(S, f^{\prime}\right)^{2} .
$$

Moreover, $|f(z)| \geq \exp \left(|z|^{\alpha}\right)$ for all $z \in S$ by Lemma 2.4, and hence

$$
f(S) \subset \mathbb{C} \backslash B_{1}=\bigcup_{S^{\prime} \in \tilde{\mathcal{S}}} S^{\prime}
$$

By (13) and Corollary 2.2, the Lebesgue measure of the union of all squares $S^{\prime} \in \tilde{\mathcal{S}} \backslash \mathcal{S}$ is at most meas $\left(E_{2}\right)<\infty$. We now consider the union of all squares $S^{\prime} \in \mathcal{S}$ with $S^{\prime} \cap$ $\partial f(S) \neq \emptyset$. The length of $\partial f(S)$ satisfies

$$
\ell(\partial f(S))=\int_{\partial S}\left|f^{\prime}(z)\right||d z| \leq \mathcal{M}\left(S, f^{\prime}\right) \ell(\partial S) \leq \frac{4 \sigma}{\sqrt{2} \mathcal{M}(S)^{d-1}} \mathcal{M}\left(S, f^{\prime}\right) .
$$

Analogously,

$$
\ell(\partial f(S)) \geq \frac{\sigma}{\sqrt{2} \mu(S)^{d-1}} \mu\left(S, f^{\prime}\right) .
$$

By Lemma 2.4, $\left|f^{\prime}(z)\right| \geq \exp \left(|z|^{\alpha}\right)$ for all $z \in S$. In particular, $\ell(\partial f(S))>1$. For $S^{\prime} \in \mathcal{S}$ with $S^{\prime} \cap \partial f(S) \neq \emptyset$, we have

$$
S^{\prime} \subset\{z: \operatorname{dist}(z, \partial f(S)) \leq 1\} .
$$

By Lemma 4.4,

$$
\operatorname{meas}(\{z: \operatorname{dist}(z, \partial f(S)) \leq 1\}) \leq \frac{9 \pi}{2} \cdot \ell(\partial f(S)) \leq \frac{18 \pi \sigma}{\sqrt{2} \mathcal{M}(S)^{d-1}} \mathcal{M}\left(S, f^{\prime}\right) .
$$

Altogether, we get

$$
\operatorname{meas}\left(f(S) \backslash \bigcup_{S^{\prime} \in \operatorname{pack}(f(S))} S^{\prime}\right) \leq \operatorname{meas}\left(E_{2}\right)+\frac{18 \pi \sigma}{\sqrt{2} \mathcal{M}(S)^{d-1}} \mathcal{M}\left(S, f^{\prime}\right) \leq \mathcal{M}\left(S, f^{\prime}\right),
$$

where we used (11) in the last step. Thus,

$$
\operatorname{dens}\left(f(S) \backslash \bigcup_{S^{\prime} \in \operatorname{pack}(f(S))} S^{\prime}, f(S)\right) \leq \frac{\mathcal{M}\left(S, f^{\prime}\right)}{\operatorname{meas}(S) \cdot \mu\left(S, f^{\prime}\right)^{2}} .
$$

Let $z_{0}$ be the centre of $S$. Then $S \subset D\left(z_{0},(\sigma / 2)\left|z_{0}\right|^{-(d-1)}\right)$, and by Lemma 3.3, $f$ is injective in $D\left(z_{0}, 2 \sigma\left|z_{0}\right|^{-(d-1)}\right)$. By the Koebe distortion theorem (Lemma 4.5), Lemma 2.4 and (12),

$$
\begin{aligned}
\operatorname{dens}\left(f(S) \backslash \bigcup_{S^{\prime} \in \operatorname{pack}(f(S))} S^{\prime}, f(S)\right) & \leq\left(\frac{5}{3}\right)^{4} \frac{1}{\operatorname{meas}(S) \mu\left(S, f^{\prime}\right)} \\
& \leq\left(\frac{5}{3}\right)^{4} \frac{32 \mu(S)^{2(d-1)}}{\sigma^{2} \exp \left(\mu(S)^{\alpha}\right)} \\
& \leq \exp \left(-\frac{1}{2} \mu(S)^{\alpha}\right) .
\end{aligned}
$$


Recall that we want to show that the density of $J_{A}$ in the square $S_{0} \in \mathcal{S}$ is large by considering the subset $T=\bigcap_{n=0}^{\infty} \bigcup_{T_{n} \in \mathcal{K}_{n}} T_{n} \subset S_{0} \cap J_{A}$. Let $n \geq 0$ and $T_{n} \in \mathcal{K}_{n}$. Then $f^{n}\left(T_{n}\right) \in \mathcal{S}$. By (14) applied to $S=f^{n}\left(T_{n}\right)$ and Lemma 2.4,

$$
\begin{aligned}
& \operatorname{dens}\left(f^{n+1}\left(T_{n}\right) \backslash \underset{S^{\prime} \in \operatorname{pack}\left(f^{n+1}\left(T_{n}\right)\right)}{\left.\bigcup^{\prime}, f^{n+1}\left(T_{n}\right)\right)}\right. \\
& \quad \leq \exp \left(-\frac{1}{2} \mu\left(f^{n}\left(T_{n}\right)\right)^{\alpha}\right) \leq \exp \left(-\frac{1}{2}\left(E_{\alpha}^{n}\left(\mu\left(S_{0}\right)\right)\right)^{\alpha}\right) .
\end{aligned}
$$

We use this to prove that the set $T_{n} \backslash\left(\bigcup_{T_{n+1} \in \mathcal{K}_{n+1}} T_{n+1}\right)$ has small density in $T_{n}$. For all $k \in\{1, \ldots, n\}$, there is a square $S_{k} \in \mathcal{S}$ such that $f^{k}\left(T_{n}\right) \subset S_{k}$. In particular, $f^{n+1}$ is injective in $T_{n}$. Thus,

$$
\begin{aligned}
& \operatorname{meas}\left(T_{n} \backslash \bigcup_{T_{n+1} \in \mathcal{K}_{n+1}} T_{n+1}\right) \\
& \leq \frac{1}{\mu\left(T_{n},\left(f^{n+1}\right)^{\prime}\right)^{2}} \cdot \operatorname{meas}\left(f^{n+1}\left(T_{n}\right) \backslash \bigcup_{S^{\prime} \in \operatorname{pack}\left(f^{n+1}\left(T_{n}\right)\right)} S^{\prime}\right),
\end{aligned}
$$

and

$$
\operatorname{meas}\left(T_{n}\right) \geq \frac{1}{\mathcal{M}\left(T_{n},\left(f^{n+1}\right)^{\prime}\right)^{2}} \operatorname{meas}\left(f^{n+1}\left(T_{n}\right)\right) .
$$

Hence,

$$
\begin{aligned}
& \operatorname{dens}\left(T_{n} \backslash \bigcup_{T_{n+1} \in \mathcal{K}_{n+1}} T_{n+1}, T_{n}\right) \\
& \leq \frac{\mathcal{M}\left(T_{n},\left(f^{n+1}\right)^{\prime}\right)^{2}}{\mu\left(T_{n},\left(f^{n+1}\right)^{\prime}\right)^{2}} \operatorname{dens}\left(f^{n+1}\left(T_{n}\right) \backslash \bigcup_{S^{\prime} \in \operatorname{pack}\left(f^{n+1}\left(T_{n}\right)\right)} S^{\prime}, f^{n+1}\left(T_{n}\right)\right) \\
& \leq\left(\frac{\mathcal{M}\left(T_{n},\left(f^{n+1}\right)^{\prime}\right)}{\mu\left(T_{n},\left(f^{n+1}\right)^{\prime}\right)}\right)^{2} \exp \left(-\frac{1}{2}\left(E_{\alpha}^{n}\left(\mu\left(S_{0}\right)\right)\right)^{\alpha}\right) .
\end{aligned}
$$

To estimate $\mathcal{M}\left(T_{n},\left(f^{n+1}\right)^{\prime}\right) / \mu\left(T_{n},\left(f^{n+1}\right)^{\prime}\right)$, let $k \in\{0, \ldots, n\}$ and $w_{0} \in f^{k}\left(T_{n}\right)$. Then

$$
f^{k}\left(T_{n}\right) \subset S_{k} \subset \overline{D\left(w_{0}, \sigma\left|w_{0}\right|^{-(d-1)}\right)} .
$$

By Lemma 3.3, $f$ is injective in $D\left(w_{0}, 2 \sigma\left|w_{0}\right|^{-(d-1)}\right)$. The Koebe distortion theorem (Lemma 4.5) yields that for all $w \in f^{k}\left(T_{n}\right)$,

$$
\frac{\left|f(w)-f\left(w_{0}\right)\right|}{\left|w-w_{0}\right|} \geq \frac{4}{9}\left|f^{\prime}\left(w_{0}\right)\right| .
$$

By Lemma 2.4, $\left|f^{\prime}\left(w_{0}\right)\right| \geq 5$. Thus,

$$
\operatorname{diam}\left(f^{k+1}\left(T_{n}\right)\right)>2 \operatorname{diam}\left(f^{k}\left(T_{n}\right)\right) .
$$


Induction yields

$$
\begin{aligned}
\operatorname{diam}\left(f^{k}\left(T_{n}\right)\right) & <\frac{1}{2^{n-k}} \operatorname{diam}\left(f^{n}\left(T_{n}\right)\right) \leq \frac{1}{2^{n-k}} \cdot \frac{\sigma}{\mathcal{M}\left(f^{n}\left(S_{0}\right)\right)^{d-1}} \\
& \leq \frac{1}{2^{n-k}} \cdot \frac{\sigma}{\mathcal{M}\left(f^{k}\left(S_{0}\right)\right)^{d-1}}
\end{aligned}
$$

In particular, for $z_{k} \in f^{k}\left(T_{n}\right)$,

$$
f^{k}\left(T_{n}\right) \subset \overline{D\left(z_{k}, \frac{2^{k-n} \sigma}{\mathcal{M}\left(f^{k}\left(S_{0}\right)\right)^{d-1}}\right)} \subset \overline{D\left(z_{k}, 2^{k-n} \sigma\left|z_{k}\right|^{-(d-1)}\right)} .
$$

Since $f$ is injective in $D\left(z_{k}, 2 \sigma\left|z_{k}\right|^{-(d-1)}\right)$, the Koebe distortion theorem (Lemma 4.5) yields

$$
\frac{\mathcal{M}\left(f^{k}\left(T_{n}\right), f^{\prime}\right)}{\mu\left(f^{k}\left(T_{n}\right), f^{\prime}\right)} \leq\left(\frac{1+2^{k-n-1}}{1-2^{k-n-1}}\right)^{4} .
$$

Since $\left(f^{n+1}\right)^{\prime}(z)=\prod_{k=0}^{n} f^{\prime}\left(f^{k}(z)\right)$, we get

$$
\begin{aligned}
\frac{\mathcal{M}\left(T_{n},\left(f^{n+1}\right)^{\prime}\right)}{\mu\left(T_{n},\left(f^{n+1}\right)^{\prime}\right)} & \leq \prod_{k=0}^{n}\left(\frac{1+2^{k-n-1}}{1-2^{k-n-1}}\right)^{4}=\left(\prod_{j=1}^{n+1} \frac{1+2^{-j}}{1-2^{-j}}\right)^{4} \\
& \leq\left(\prod_{j=1}^{\infty} \frac{1+2^{-j}}{1-2^{-j}}\right)^{4}=: C_{2}
\end{aligned}
$$

where $C_{2} \in(0, \infty)$. Together with (15), this implies

$$
\operatorname{dens}\left(T_{n} \backslash \bigcup_{T_{n+1} \in \mathcal{K}_{n+1}} T_{n+1}, T_{n}\right) \leq C_{2}^{2} \exp \left(-\frac{1}{2}\left(E_{\alpha}^{n}\left(\mu\left(S_{0}\right)\right)\right)^{\alpha}\right) .
$$

Thus,

$$
\begin{aligned}
\operatorname{meas}\left(S_{0} \backslash T\right) & =\sum_{n=0}^{\infty} \sum_{T_{n} \in \mathcal{K}_{n}} \operatorname{meas}\left(T_{n} \backslash \bigcup_{T_{n+1} \in \mathcal{K}_{n+1}} T_{n+1}\right) \\
& =\sum_{n=0}^{\infty} \sum_{T_{n} \in \mathcal{K}_{n}} \operatorname{dens}\left(T_{n} \backslash \bigcup_{T_{n+1} \in \mathcal{K}_{n+1}} T_{n+1}, T_{n}\right) \cdot \operatorname{meas}\left(T_{n}\right) \\
& \leq \sum_{n=0}^{\infty} C_{2}^{2} \exp \left(-\frac{1}{2}\left(E_{\alpha}^{n}\left(\mu\left(S_{0}\right)\right)\right)^{\alpha}\right) \sum_{T_{n} \in \mathcal{K}_{n}} \operatorname{meas}\left(T_{n}\right) \\
& \leq C_{2}^{2} \sum_{n=0}^{\infty} \exp \left(-\frac{1}{2}\left(E_{\alpha}^{n}\left(\mu\left(S_{0}\right)\right)\right)^{\alpha}\right) \cdot \operatorname{meas}\left(S_{0}\right) .
\end{aligned}
$$

For all large $x, \exp \left(\alpha x^{\alpha}\right) \geq x^{\alpha}+2 \log 2$, and thus

$$
\exp \left(-\frac{1}{2}\left(E_{\alpha}(x)\right)^{\alpha}\right)=\exp \left(-\frac{1}{2} \exp \left(\alpha x^{\alpha}\right)\right) \leq \frac{1}{2} \exp \left(-\frac{1}{2} x^{\alpha}\right) .
$$


Induction yields

$$
\sum_{n=0}^{\infty} \exp \left(-\frac{1}{2}\left(E_{\alpha}^{n}\left(\mu\left(S_{0}\right)\right)\right)^{\alpha}\right) \leq \sum_{n=0}^{\infty} \frac{1}{2^{n}} \exp \left(-\frac{1}{2} \mu\left(S_{0}\right)^{\alpha}\right)=2 \exp \left(-\frac{1}{2} \mu\left(S_{0}\right)^{\alpha}\right)
$$

Thus,

$$
\operatorname{meas}\left(S_{0} \backslash J_{A}\right) \leq \operatorname{meas}\left(S_{0} \backslash T\right) \leq 2 C_{2}^{2} \exp \left(-\frac{1}{2} \mu\left(S_{0}\right)^{\alpha}\right) \operatorname{meas}\left(S_{0}\right) .
$$

To conclude that meas $\left(\mathbb{C} \backslash J_{A}\right)<\infty$, fix $R>0$ such that $B_{0} \subset D(0, R / 2)$. For $r \geq R$, define

$$
\operatorname{ann}(r):=\{z: r \leq|z| \leq 2 r\}
$$

Then

$$
\bigcup_{\substack{S_{0} \in \mathcal{S} \\ S_{0} \cap \operatorname{ann}(r) \neq \emptyset}} S_{0} \subset\left\{z: \frac{r}{2} \leq|z| \leq 3 r\right\} \subset \overline{D(0,3 r)} .
$$

We get

$$
\begin{aligned}
& \operatorname{meas}\left(\operatorname{ann}(r) \backslash\left(J_{A} \cup E_{2}\right)\right) \leq \sum_{\substack{S_{0} \in \mathcal{S} \\
S_{0} \cap \operatorname{ann}(r) \neq \emptyset}} 2 C_{2}^{2} \exp \left(-\frac{1}{2} \mu\left(S_{0}\right)^{\alpha}\right) \operatorname{meas}\left(S_{0}\right) \\
& \leq 2 C_{2}^{2} \exp \left(-\frac{1}{2}\left(\frac{r}{2}\right)^{\alpha}\right) \sum_{\substack{S_{0} \in \mathcal{S} \\
S_{0} \cap \operatorname{nann}(r) \neq \emptyset}} \operatorname{meas}\left(S_{0}\right) \leq 2 C_{2}^{2} \exp \left(-\frac{r^{\alpha}}{2^{1+\alpha}}\right) \operatorname{meas}(D(0,3 r)) \\
& =18 \pi C_{2}^{2} r^{2} \exp \left(-\frac{r^{\alpha}}{2^{1+\alpha}}\right) \leq \exp \left(-\frac{r^{\alpha}}{2^{2+\alpha}}\right)
\end{aligned}
$$

if $r$ is sufficiently large. Applying this to the annuli ann $\left(2^{n} R\right), n \geq 0$, yields

$$
\begin{aligned}
\operatorname{meas}\left(\mathbb{C} \backslash J_{A}\right) & \leq \operatorname{meas}(D(0, R))+\operatorname{meas}\left(E_{2}\right)+\sum_{n=0}^{\infty} \operatorname{meas}\left(\operatorname{ann}\left(2^{n} R\right) \backslash\left(J_{A} \cup E_{2}\right)\right) \\
& \leq \operatorname{meas}(D(0, R))+\operatorname{meas}\left(E_{2}\right)+\sum_{n=0}^{\infty} \exp \left(-\frac{R^{\alpha}}{2^{2+\alpha}} 2^{n \alpha}\right)<\infty
\end{aligned}
$$

This completes the proof.

5. Verification of the properties of Examples 1.1 and 1.3

We first verify the properties of Example 1.1, that is, we prove that the Fatou set and the non-escaping set of $\sin (z)$ and $\sin \left(z^{2}\right)$ have infinite Lebesgue measure.

First, let $f(z)=\sin (z)$. From $f(0)=0$ and $f^{\prime}(0)=1$, it follows that $F(f) \neq \emptyset$, and that there exists a component of $F(f)$ where $\left(f^{n}(z)\right)$ tends to zero. Since $F(f)$ is open and $2 \pi$-periodic, the Lebesgue measure of $F(f)$ and $\mathbb{C} \backslash I(f)$ is infinite.

Let us now consider the function $f(z)=\sin \left(z^{2}\right)$. Note that $f$ has a superattracting fixed point at zero. Let $\varepsilon>0$ such that $D(0, \varepsilon)$ is contained in the attractive basin of zero. There 
exists $\delta \in(0, \pi / 2)$ such that $\sin (D(\pi k, \delta)) \subset D(0, \varepsilon)$ for all $k \in \mathbb{Z}$. Let $D_{k}:=D(\pi k, \delta)$ and $p(z)=z^{2}$. Then $p^{-1}\left(D_{k}\right)$ is contained in the attractive basin of zero of the function $f$. We get

$$
\operatorname{meas}\left(p^{-1}\left(D_{k}\right)\right)=2 \int_{D_{k}}\left(\frac{1}{2 \sqrt{|z|}}\right)^{2} d x d y \geq \frac{1}{2(|k| \pi+\delta)} \operatorname{meas}\left(D_{k}\right)=\frac{\pi \delta^{2}}{2(|k| \pi+\delta)} .
$$

Summing up over all $k$ yields that the attractive basin of zero has infinite measure.

In the remaining part of this section, we consider the function

$$
h(z)=\frac{1}{2} \exp \left(z^{3}+i z\right)-\frac{1}{2} \exp \left(-z^{3}+i z\right)=\exp (i z) \sinh \left(z^{3}\right)
$$

given in Example 1.3. Note that $h$ satisfies all assumptions of Theorem 1.4 except for condition (3). Moreover, function $h$ has a superattracting fixed point at zero. Recall that we want to prove that the attractive basin of zero has infinite Lebesgue measure, so that in particular the Lebesgue measure of $\mathbb{C} \backslash(J(h) \cap A(h))$ is infinite.

To do so, fix $\varepsilon>0$ such that $D(0, \varepsilon)$ is contained in the attractive basin of zero. For large $r_{0}>1$, let

$$
B:=\left\{r e^{i \theta}: r \geq r_{0},\left|\theta-\frac{\pi}{2}\right| \leq \frac{1}{r^{2} \log r}\right\}
$$

We have

$$
\operatorname{meas}(B)=\int_{r_{0}}^{\infty} \int_{\pi / 2-1 /\left(r^{2} \log r\right)}^{\pi / 2+1 /\left(r^{2} \log r\right)} r d \theta d r=\int_{r_{0}}^{\infty} \frac{2}{r \log r} d r=2 \int_{\log \left(r_{0}\right)}^{\infty} \frac{1}{u} d u=\infty .
$$

We now show that if $r_{0}$ is sufficiently large, then $h(B) \subset D(0, \varepsilon)$, and hence $B$ is contained in the attractive basin of zero. Let $z=r e^{i \theta} \in B$. Then

$$
\begin{aligned}
|h(z)| & \leq|\exp (i z)| \cdot \frac{1}{2}\left(\left|\exp \left(z^{3}\right)\right|+\left|\exp \left(-z^{3}\right)\right|\right) \\
& =\exp (-r \sin (\theta)) \cdot \frac{1}{2}\left(\exp \left(r^{3} \cos (3 \theta)\right)+\exp \left(-r^{3} \cos (3 \theta)\right)\right) \\
& \leq \exp (-r \sin (\theta)) \cdot \exp \left(r^{3}|\cos (3 \theta)|\right) .
\end{aligned}
$$

We have

$$
\sin (w)=1+O\left(\left(w-\frac{\pi}{2}\right)^{2}\right) \quad \text { as } w \rightarrow \frac{\pi}{2}
$$

and

$$
\cos (w)=\left(w-\frac{3 \pi}{2}\right)+\mathcal{O}\left(\left(w-\frac{3 \pi}{2}\right)^{3}\right) \quad \text { as } w \rightarrow \frac{3 \pi}{2} .
$$

Since $|\theta-\pi / 2| \leq 1 /\left(r^{2} \log r\right)$, this implies

$$
-\sin (\theta) \leq-\frac{1}{2}
$$

and

$$
|\cos (3 \theta)| \leq 2 \cdot \frac{3}{r^{2} \log r}
$$


if $r$ is sufficiently large. Thus,

$$
|h(z)| \leq \exp \left(-\frac{1}{2} r\right) \cdot \exp \left(6 \cdot \frac{r}{\log r}\right) \leq \exp \left(-\frac{1}{4} r\right)<\varepsilon
$$

if $r$ is sufficiently large. So $h(B) \subset D(0, \varepsilon) \subset F(h) \backslash I(h)$ if $r_{0}$ is sufficiently large.

Acknowledgements. I would like to thank Walter Bergweiler for helpful suggestions. Moreover, I would like to thank the referee for detailed and valuable comments.

\section{REFERENCES}

[1] M. Aspenberg and W. Bergweiler. Entire functions with Julia sets of positive measure. Math. Ann. 352(1) (2012), 27-54.

[2] W. Bergweiler. Iteration of meromorphic functions. Bull. Amer. Math. Soc. (N. S.) 29(2) (1993), 151-188.

[3] W. Bergweiler. Lebesgue measure of Julia sets and escaping sets of certain entire functions. Fund. Math. 242(3) (2018), 281-301.

[4] W. Bergweiler and I. Chyzhykov. Lebesgue measure of escaping sets of entire functions of completely regular growth. J. Lond. Math. Soc. (2) 94(2) (2016), 639-661.

[5] W. Bergweiler and A. Hinkkanen. On semiconjugation of entire functions. Math. Proc. Cambridge Philos. Soc. 126(3) (1999), 565-574.

[6] H. Bock. On the dynamics of entire functions on the Julia set. Results Math. 30(1-2) (1996), 16-20.

[7] A. E. Eremenko. On the iteration of entire functions. Dynamical Systems and Ergodic Theory (Warsaw 1986) (Banach Center Publications, 23). PWN, Warsaw, 1989, pp. 339-345.

[8] J.-M. Hemke. Recurrence of entire transcendental functions with simple post-singular sets. Fund. Math. 187(3) (2005), 255-289.

[9] C. McMullen. Area and Hausdorff dimension of Julia sets of entire functions. Trans. Amer. Math. Soc. 300(1) (1987), 329-342.

[10] C. Pommerenke. Univalent Functions (Studia Mathematica/Mathematische Lehrbücher, XXV). Vandenhoeck \& Ruprecht, Göttingen, 1975.

[11] C. Pommerenke. Boundary Behaviour of Conformal Maps (Grundlehren der Mathematischen Wissenschaften, 299). Springer, Berlin, 1992.

[12] P. J. Rippon and G. M. Stallard. Fast escaping points of entire functions. Proc. Lond. Math. Soc. (3) 105(4) (2012), 787-820.

[13] H. Schubert. Area of Fatou sets of trigonometric functions. Proc. Amer. Math. Soc. 136(4) (2008), 1251-1259.

[14] D. J. Sixsmith. Julia and escaping set spiders' webs of positive area. Int. Math. Res. Not. 2015(19) (2015), 9751-9774.

[15] S. Zhang and F. Yang. Area of the complement of the fast escaping set of a family of entire functions. Kodai Math. J. 41(3) (2018), 531-553.

[16] J.-H. Zheng. On multiply-connected Fatou components in iteration of meromorphic functions. J. Math. Anal. Appl. 313(1) (2006), 24-37. 